haemoglobin fraction in the haemolysate may be due to its early intracellular precipitation. ${ }^{2}$ This applies to the surplus of $\alpha$-chains in $\beta$-thalassaemias, including the $\beta$-thalassaemia/ $\mathrm{Hb}$ E association ${ }^{3}$; it holds in part for the surplus of $\beta$-chains in $\alpha$-thalassaemia intermedia. " I suggest that in $\mathrm{Hb} \mathrm{E} / \alpha$-thalassaemia the expected excess of $\beta^{\mathrm{B}}$ chains precipitates intracellularly. Indeed, Dr. Wasi and his colleagues observed a small percentage of red cells with inclusion bodies, which they attributed to $\mathrm{Hb}_{4}^{\mathrm{A}}$. It is not stated whether the inclusions were preformed in vivo or had developed in vitro. It is more probable that the precipitates are composed mainly, though not of necessity exclusively, of $\beta^{\mathrm{E}}$ chains and that higher proportions of inclusions occur in red cell precursors. A low inclusion cell count in the circulation does not necessarily imply a minor degree of haemoglobin precipitation. On the contrary, it may be due to early and extensive precipitation, leading to rapid selective removal of inclusions and/or affected cells and to enrichment in cells containing $\mathrm{Hb}_{\mathfrak{s}}$, which is the most stable tetramer of this class. If $\beta^{\mathbb{B}}$ chains are less stable than $\beta^{\mathbf{A}}$ increasingly more $\beta^{\mathbf{A}}$ chains will be available to combine with the short supply of $\alpha$-chains and the $A / E$ ratio will rise. Perhaps one should expect a series of tetrameric molecules $\beta_{4}^{\mathbb{R}}, \beta_{3}^{\mathbb{R}}, \beta_{1}^{\mathbf{A}}, \beta_{2}^{\mathbb{R}}, \beta_{2}^{\mathbf{A}}, \beta_{1}^{\mathbf{R}}$, $\beta_{3}^{A}$, and $\beta_{4}^{A}$, in order of increasing stability; lability of these molecules will lead to some loss of $\beta^{\mathbf{A}}$ chains as well, so that finally only minute quanties of free $\beta^{\mathbf{A}}$ will be left over.

The suggested preferential uptake of $\alpha$-chains by $\beta^{A}$ relative to $\beta^{B}$ also may contribute to an increase of the $A / E$ ratio." However, in the relevant hybridization experiment $\beta^{A}$ chains outnumbered the $\beta^{\mathbb{E}}$ chains from the start by a factor of four. Were competition solely responsible for the high $A / E$ ratio, one would expect a large surplus of $\beta^{E}$ chains.

Finally, greater instability of $\beta^{\mathbb{E}}$ compared to $\beta^{A}$ is compatible with the fact that $\alpha$-chain deficiency combined with $\mathrm{Hb} \mathbf{E}$ is more severe, clinically and haematologically, than classical $\mathbf{H b} \mathbf{H}$ disease, although the imbalance of chain synthesis is more obvious in the latter. $-\mathrm{I} \mathrm{am}$, etc.,

Phaedon Fessas.

Aematology Section and Laboratory,

Alexandra Hospital,
Athens, Greece.

REPERBNCES

- Tuchinda, S., Rucknagel, D. L., Minnich, V., Boonyaprakob, $U$., Balankura, $\mathrm{K}$., and Suvate

V., American \}. hum. Genet., 1964, 16, 311.

- Fessas, Ph. Intern. Symposium on Comparative
Haemoglobin Structure, Thessaloniki, 1966, p.

- Fessas, Ph., Blood, 1963, 21, 21. . Pessas, Ph., and Yataghanas, X., Blood, 1968, in

the press. S., Beale, D., and Lehmann, H.
Tuchinda,
Human Geretik, 1967, 3, 312.

\section{Volvulus of the Sigmoid Colon}

SIR,-The review of 425 cases from Mulago Hospital, Kampala, Uganda, by Mr. J. J. Shepherd (3 February, p. 280) during the period 1949-65 suggests a higher incidence in Uganda than in the part of India from which I reported 40 cases during a fiveyear period in 1956.' However, to establish this I suggest it would be valuable if the actual percentage of volvulus cases to all surgical inpatients could be given, so that a basis of comparison is established. The rate at the Evangeline Booth Hospital during the years 1948-53 was about $1.5 \%$ of the surgical admissions for pelvic colon volvulus and $2.5 \%$ for all varieties of volvulus.

I recently had the opportunity in Norway of studying the rate of hospital incidence in surgical patients at the Ulleval Hospital, Oslo, for common abdominal surgical diseases from 1911 to 1960. " The "time trends " in the five diseases studied-namely, appendicitis, urolithiasis, cholecystitis and cholelithiasis, inguinal hernia, and volvulus-

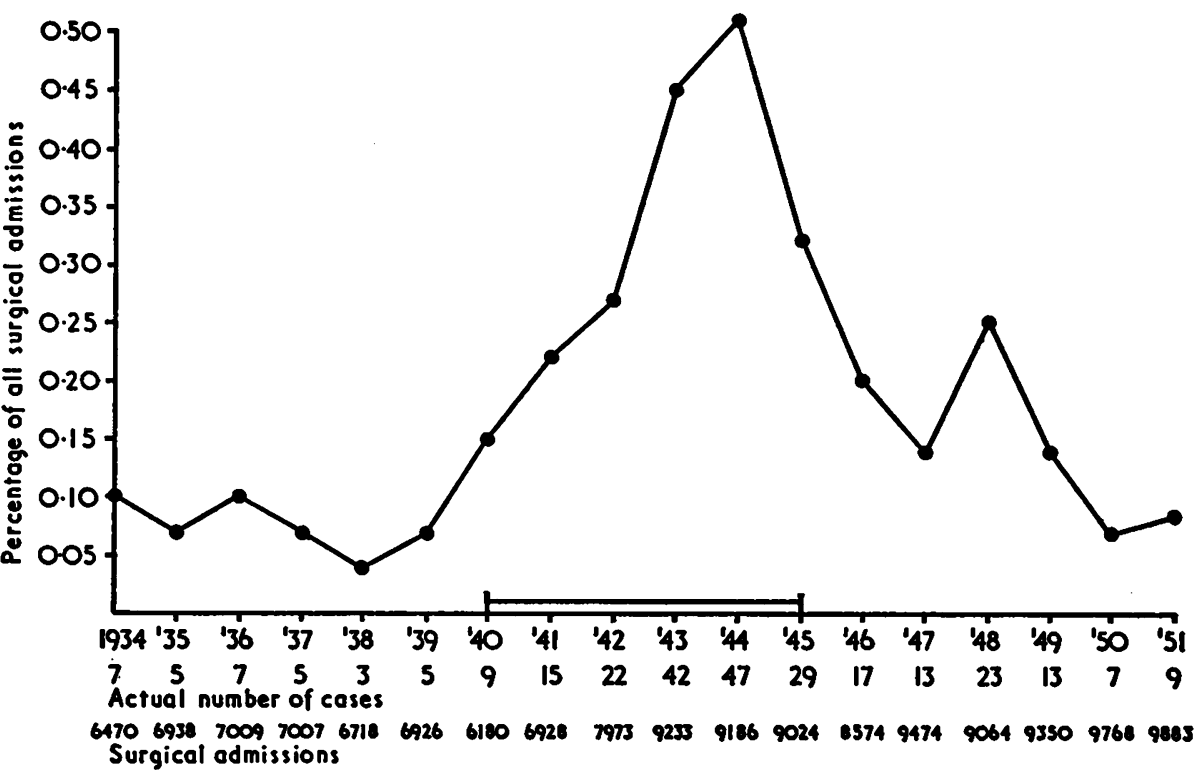

were found to be similar in pattern, although with local variations, in 10 other Norwegian hospitals from 1921 to 1960 , so far as records were available, and the pattern in appendicitis, urolithiasis, and cholelithiasis was also found to have the same "time trend " in the insured population incidence in the City of Oslo 1940-60. This seems to indicate that the hospital incidence figures can be regarded as a fairly reliable guide to the population incidence of the diseases studied when the incidence figures are obtained from a general hospital serving a wide area. The rates of incidence in the Oslo City Hospital showed a remarkably interesting relationship to both the first and the second world war years, which has already been pointed out. ${ }^{2-5}$ Volvulus was relatively common before and during the first world war, having an incidence of about $0.9 \%$ of the total surgical inpatients. It fell to under $0.1 \%$ between the two world wars, but

\section{Neurosyphilis}

SIR,-I was interested to read the paper on the changing clinical picture of neurosyphilis by Dr. R. Joffe and colleagues (27 January, p. 211). Since June 1964 I have kept a spare copy of all letters filed according to the diagnosis. During this period up until January 1968 I have seen 30 cases of neurosyphilis. The various types of disease were as follows:

Tabes dorsalis

Spinal mening $\quad \ldots \quad \ldots \quad 10$ cases Cerebral meningovascular syphilis .... 13 cases Cerebral meningosyphilis with epilepsy 2 " General paralysis of insane $\ldots . \ldots .3$

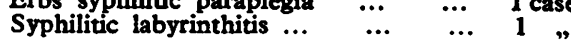

rose gradually from 1940 to reach a peak in 1944 of about $0.51 \%$ and then subsided gradually over the next six years to return to a level of about $0.09 \%$, as shown in the graph. The figures from six other hospitals in southern Norway which provided information about cases of volvulus showed a similar rise during the war, but this rise was not observed in two hospitals in northern Norway, which had a low level throughout.

These observations strengthen the sugges tion made by Bruusgaard ${ }^{3}$ that a compulsory

change of dietary habits during both world wars, from a mixed to a mainly coarser vegetarian one, had an aetiological relationship to the condition, possibly affecting a few of that proportion of the population with a congenitally long or otherwise abnormal pelvic colon. This supports the earlier observations made in India, and suggests the usefulness of further incidence studies in relation to our dietary habits and structure which could point towards remedial measures.-I am, etc.

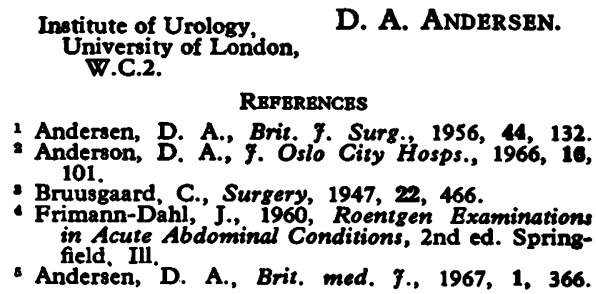

1 Andersen, D. A., Brit. F. Surg. 1956, 14, 132. 101 .

- Bruusgaard, C., Surgery, 1947, 22, 466.

rimann-Dahl, J., 1960, Roentgen Examination in Acute Abdominal Conditions, 2 nd ed. Spring-

s Andersen, D. A., Brit. med. 7., 1967, 1, 366.

These figures are obtained from the neurological clinics of three major hospitals in the Greater London area and one in Bedfordshire, although I left Harold Wood Hospital in March 1966. Fourteen patients were attending Whipps Cross Hospital, 10 Luton and Dunstable Hospital, 4 Barnet General, and 2 Harold Wood Hospital.

The figures are comparable to those of the Newcastle series: 7 cases in a period of nine months-and the catchment population area is very similar $(900,000)$. As in the Newcastle area, several presented difficult diagnostic problems, and one patient had a familial muscular dystrophy as well as signs of a spastic paraplegia and Argyll Robertson 